\title{
Imaging Biomarkers in Thoracic Oncology: Current Advances in the Use of Radiomics in Lung Cancer Patients and its Potential Use for Therapy Response Prediction and Monitoring
}

\section{Imaging Biomarker in der Thoraxonkologie: Aktuelle Fortschritte im Einsatz von Radiomics bei Lungenkrebspatienten und die Möglichkeit von Therapievorhersage und Verlaufskontrolle}

Authors

Jonas Kroschke, 2, 3@, Oyunbileg von Stackelberg 1, 2, 3, Claus Peter Heußel 1, 2, 3, Mark Oliver Wielpütz ${ }^{1,2,3}$, Hans-Ulrich Kauczor ${ }^{1,2,3}$

Affiliations

1 Department for Diagnostic and Interventional Radiology, University Hospital Heidelberg, Germany

2 Translational Lung Research Center (TLRC), German Center for Lung Research, Giessen, Germany

3 Department for Diagnostic and Interventional Radiology with Nuclear Medicine, Thoraxklinik am Universitätsklinikum Heidelberg, Germany

Key words

radiomics, artificial intelligence, imaging biomarkers, non-small cell lung cancer, tumor heterogeneity

received 30.04.2021

accepted 20.12.2021

published online 24.02 .2022

Bibliography

Fortschr Röntgenstr 2022; 194: 720-727

DOI 10.1055/a-1729-1516

ISSN 1438-9029

(C) 2022. Thieme. All rights reserved.

Georg Thieme Verlag KG, Rüdigerstraße 14,

70469 Stuttgart, Germany

Correspondence

Herr Dr. Jonas Kroschke

Diagnostic and Interventional Radiology, University Hospital Heidelberg, Im Neuenheimer Feld 420, 69120 Heidelberg,

Germany

Tel.: +49/6221/5632865

j.kroschke@icloud.com

\section{ABSTRACT}

Background Non-small cell lung cancer (NSCLC) is the leading cause of cancer-related deaths. The development of therapies targeting molecular alterations has significantly improved the treatment of NSCLC patients. To identify these targets, tumor phenotyping is required, with tissue biopsies and molecular pathology being the gold standard. Some patients do not respond to targeted therapies and many patients suffer from tumor recurrence, which can in part be explained by tumor heterogeneity. This points out the need for new biomarkers allowing for better tumor phenotyping and monitoring during treatment to assess patient outcome. Method The contents of this review are based on a literature search conducted using the PubMed database in March 2021 and the authors' experience.

Results and Conclusion The use of radiomics and artificial intelligence-based approaches allows for the identification of imaging biomarkers in NSCLC patients for tumor phenotyping. Several studies show promising results for models predicting molecular alterations, with the best results being achieved by combining structural and functional imaging. Radiomics could help solve the pressing clinical need for assessing and predicting therapy response. To reach this goal, advanced tumor phenotyping, considering tumor heterogeneity, is required. This could be achieved by integrating structural and functional imaging biomarkers with clinical data sources, such as liquid biopsy results. However, to allow for radiomics-based approaches to be introduced into clinical practice, further standardization using large, multi-center datasets is required.

Key points:

- Some NSCLC patients do not benefit from targeted therapies, and many patients suffer from tumor recurrence, pointing out the need for new biomarkers allowing for better tumor phenotyping and monitoring during treatment.

- The use of radiomics-based approaches allows for the identification of imaging biomarkers in NSCLC patients for tumor phenotyping.

- A multi-omics approach integrating not only structural and functional imaging biomarkers but also clinical data sources, such as liquid biopsy results, could further enhance the prediction and assessment of therapy response.

\section{Citation Format}

- Kroschke J, von Stackelberg O, Heußel CP et al. Imaging Biomarkers in Thoracic Oncology: Current Advances in the Use of Radiomics in Lung Cancer Patients and its Potential 
Use for Therapy Response Prediction and Monitoring. Fortschr Röntgenstr 2022; 194: 720-727

\section{ZUSAMMENFASSUNG}

Hintergrund Nichtkleinzelliger Lungenkrebs ist die häufigste Krebstodesursache. Durch die Entwicklung neuer gezielter Therapien konnten erhebliche Fortschritte in der Behandlung von Lungenkrebspatienten erzielt werden. Gewebebiopsien und molekulare Pathologie sind der Goldstandard zur Identifikation der molekularen Therapieziele. Nicht alle Patienten profitieren von diesen neuen Therapieformen und viele Patienten leiden an Rezidiven, was z. T. durch die Tumorheterogenität erklärt werden kann. Die Identifikation weiterer Biomarker ist nötig, um das Therapieansprechen besser beurteilen zu können.

Methode Der Inhalt dieser Übersichtarbeit basiert auf einer Literaturrecherche in PubMed aus dem März 2021 und den Erfahrungen der Autoren.

Ergebnisse und Schlussfolgerung Mithilfe von Radiomics und maschinellem Lernen können Bildgebungsbiomarker zur Tumorphänotypisierung bei Lungenkrebspatienten identifiziert werden. Einige Studien zeigen gute Ergebnisse der Prädiktionsmodelle für das Vorliegen verschiedener molekularer Alterationen, wobei die Verwendung von struktureller und funktionaler Bildgebung die besten Ergebnisse liefert. Durch die Integration struktureller und funktionaler Bildgebung mit weiteren klinischen Datenquellen, wie den Ergebnissen von liquid biopsies, könnte Radiomics ein Lösungsansatz für die klinische Notwendigkeit zur besseren Beurteilung des Therapieverlaufs sein. Damit Radiomics-Ansätze Einzug in die klinische Routine halten können, sind weitere Studien mit großen, multizentrischen Datensätzen zur Validierung nötig.

\section{Kernaussagen:}

- Nicht alle Patienten mit nichtkleinzelligem Lungenkrebs profitieren von gezielten Therapien und viele Patienten entwickeln Rezidive, was die Notwendigkeit der Identifikation neuer Biomarker zur besseren Phänotypisierung und Verlaufskontrolle von Tumoren aufzeigt.

- Mithilfe von Radiomics können Bildgebungsbiomarker zur Tumorphänotypisierung bei Lungenkrebspatienten identifiziert werden.

- Durch die Integration struktureller und funktionaler Bildgebung mit weiteren klinischen Datenquellen, wie den Ergebnissen von liquid biopsies, könnte Radiomics ein Lösungsansatz für die klinische Notwendigkeit zur besseren Beurteilung des Therapieverlaufs sein.

\section{Introduction}

The field of radiology has undergone substantial changes due to technological advances with the introduction of computed tomography (CT) and magnetic resonance imaging (MRI) and their subsequent steady improvement, which accounts for the major changes in diagnostic medicine and treatment response measures in the field of oncology. The next step in the innovation of radiology will be mainly due to the advances made in the application of computer-assisted analysis of imaging and clinical data, especially radiomics and artificial intelligence. Radiological imaging plays a major role in the clinical decision-making process in oncology, and computer-assisted image analysis promises to offer obvious improvement to this process where the human expert fails to comprehend the complex information beyond tumor size and contrast behavior.

Radiological imaging in lung cancer patients is one of the fields in oncology in which such applications are eagerly awaited. Lung cancer is the second most common cancer type globally and the leading cause of cancer deaths, with non-small cell lung cancer (NSCLC) being the predominant subtype, accounting for approximately $85 \%$ of cases with approximately $40 \%$ being classified as adenocarcinomas [1, 2]. Early detection of malignant pulmonary lesions is highly relevant, as the 5 -year survival rate of patients treated with early stage lung cancer is $57 \%$, while it is only about $5 \%$ in patients with generalized disease [3]. Due to limited symptoms in early stages, lung cancer is often diagnosed in late stages [4]. In recent years the identification of new molecular and genomic biomarkers has provided new targets for therapeutic approa- ches in patients with late-stage non-small cell lung cancer (NSCLC), thereby increasing overall survival [5, 6]. Important examples include mutations of the epidermal growth factor receptor (EGFR) or anaplastic lymphoma kinase (ALK) rearrangements, which can be found in $15 \%$ and $2 \%$ of non-small cell lung cancer patients, respectively $[7,8]$. For both alterations a number of targeted therapeutics are already being used in the clinical routine (e.g., the tyrosine kinase inhibitors erlotinib, gefitinib, and afatinib for EGFR mutations [9, 10] and crizotinib, alectinib, and brigatinib for ALK translocations [11, 12]). The histological subtypes of lung cancer and molecular alterations in the biggest histological subgroup, i. e., adenocarcinomas, are summarized in $\mathbf{~ F i g . ~} \mathbf{1}$

To date, the identification of the aforesaid mutations usually requires the invasive collection of tissue samples, e.g., by transbronchial biopsy or CT-guided biopsy of the primary tumor or its metastases. Invasive procedures carry the risk of complications and consequently not all patients can undergo these procedures due to comorbidities. In some cases, tissue samples might be inadequate or in the case of oncological progression re-biopsy cannot always be performed. To overcome these hurdles, new approaches are being investigated to assess and monitor tumor mutation status, for example the examination of circulating cellfree nucleic acids in the blood stream, usually referred to as liquid biopsy [13], or the use of surrogate markers as derived, e. g., from imaging through computer-assisted analysis of radiological imaging or clinical data. Even though the identification of the described molecular biomarkers as therapy targets has had a considerable impact on patient outcome, not all patients with corresponding molecular alterations benefit from targeted thera- 


\section{Subtypes of lung cancer}

\section{Molecular alterations in lung adenocarcinoma}
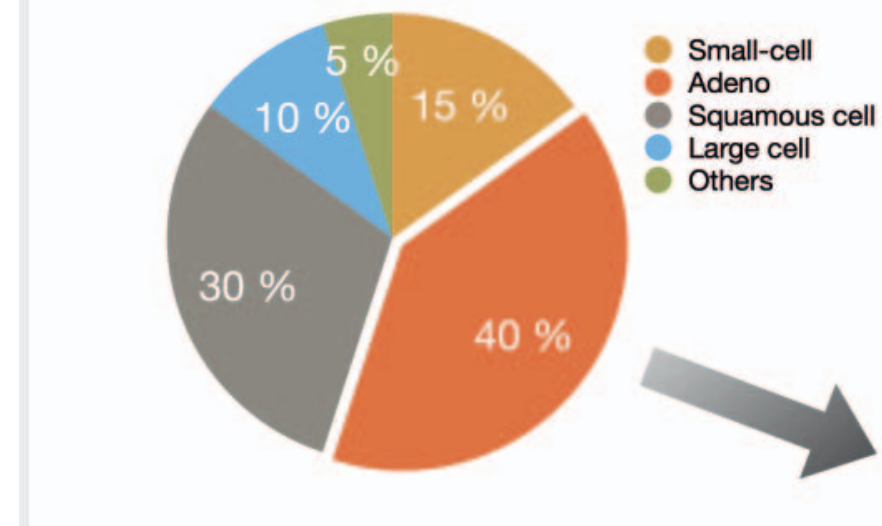 \\ Fig. 1 Overview of histologica
adenocarcinomas (right) [1]. dem Adenokarzinom (rechts) [1]. \\ pies and in many cases tumor recurrence is observed over time, highlighting the need for close monitoring of therapy response as part of the diagnostic process in lung cancer patients. In this review we give a short introduction to the field of radiomics in the context of identification of imaging biomarkers in lung cancer and provide an outlook on how radiomics could impact the management and treatment of lung cancer patients in the future. \\ Basic Principles of Radiomics for the Identification of Imaging Biomarkers}

Unknown: $230 \%$

- Abb. 1 Übersicht der histologischen Subtypen von Lungenkrebs (links) und der molekularen Alterationen der größten Subgruppe,

In oncologic radiology the evaluation of imaging traditionally involves a mainly qualitative approach by the human reader, known as semantic [14]. This refers to cross-sectional imaging, both CT and MRI. The tumor phenotype can only be partially detected by the human eye, also depending on factors like the experience of the radiologist. Quantitative analysis in this context is often limited to one-dimensional measurements of tumor manifestations with follow-up examinations being evaluated according to RECIST (response evaluation of criteria for solid tumors) guidelines [15]. This approach potentially fails to recognize a large part of the information available from imaging, as this information might not be easily accessible to the human eye. Fig. 2 shows three examples of pulmonary adenocarcinomas with a different tumor mutation status, showing no obvious features that make it possible to differentiate them visually.

An increasing number of studies show that radiomics-based image analysis allows for the extraction of otherwise missed features and their quantitative analysis [16], which in turn could improve diagnosis and might lead to a better prediction of tumor response to therapy [17]. The term radiomics refers to the concept of large-scale analysis of radiological images and the association with biological markers or clinical endpoints using mathematical and machine learning methods [18]. The main steps of any radiomics workflow follow the same principles and can be summarized as shown in > Fig. 3 .

Image acquisition is the starting point in any radiological study. Differences in imaged organ systems and image acquisition techniques can have a considerable impact on the reproducibility of radiomics models as scanners, scanning protocols, reconstruction algorithms, etc. can vary significantly in large data sets [19]. As most radiomic analyses are conducted retrospectively, this factor must be considered and needs to be addressed by techniques like feature normalization or harmonization $[20,21]$. Otherwise, the resulting model might be limited by trained technical pre-settings. Following image acquisition, the region of interest (ROI) needs to be defined and segmented for further analysis. This may not only include the tumor itself but also its close pulmonary vicinity to study the interaction with surrounding tissues. Semiautomated and fully automated segmentation techniques have improved in recent years, speeding up the otherwise time-consuming work of manual segmentation by experts [22].

Radiomic features are consequently calculated from the segmented structures. Many different features have been described, some being highly standardized while other studies also include handcrafted features. Authors take different approaches in the 

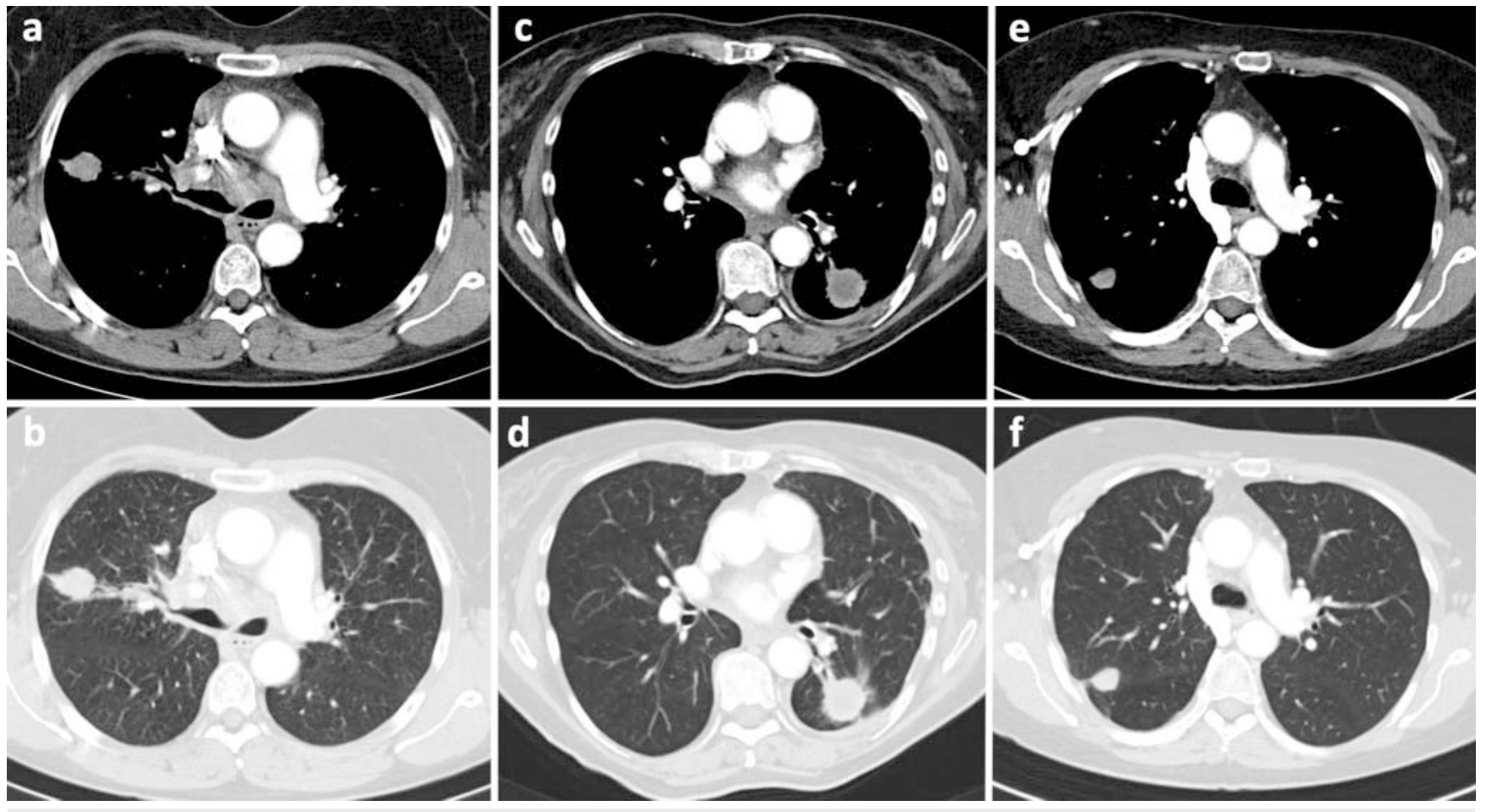

- Fig. 2 Examples of pulmonary adenocarcinomas in soft tissue (a, c, e) and lung tissue window (b, d, f): CT examinations of three different patients at the time of initial diagnosis. Patients later underwent tissue biopsies with molecular pathological analysis detecting no tumor mutation (a, b), EGFR mutation (c, d), and ALK rearrangement (e, f).

- Abb. 2 Beispiele pulmonaler Adenokarzinome im Weichteil- (a, c, e) und Lungenfenster (b, d, f): CT-Untersuchungen von 3 unterschiedlichen Patienten zum Zeitpunkt der Erstdiagnose, bei denen später eine Biopsie mit anschließender Molekularpathologie durchgeführt wurde. Es zeigten sich keine Mutation (a, b), eine EGFR-Mutation (c, d) und eine ALK-Translokation (e, f).
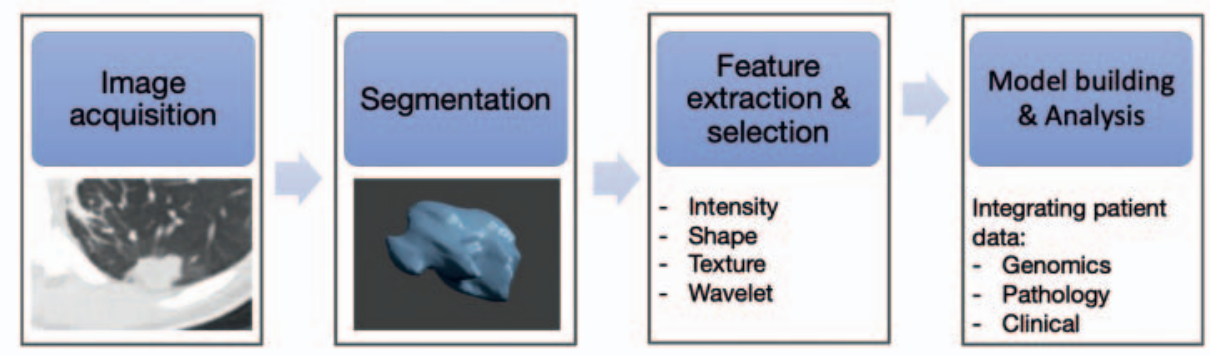

- Fig. 3 Steps of radiomics workflows: After image acquisition, target structures are either manually or (semi-)automatically segmented for the feature extraction and selection process, which is succeeded by analysis of the association of radiomics features with other endpoints to establish a prediction mode, followed by performance testing of the established model.

- Abb. 3 Schritte eines Radiomics-Arbeitsflusses: Nach der Bildakquisition werden die Zielstrukturen für den Extraktions- und Selektionsprozess der Bildeigenschaften manuell oder (semi-)automatisch segmentiert, gefolgt von der Analyse der Assoziation der erhobenen Bildeigenschaften mit definierten Endpunkten zur Entwicklung eines Modells, welches im letzten Schritt getestet werden muss.

classification of features. Conventionally, four main groups can be distinguished: tumor intensity-based features (also called first order features as first-order statistics are used for description), shape features, texture features and wavelet features. Tumor intensity-based features are histogram-based quantifications of all tumor voxel intensity values. Shape features describe the geometric properties of the region of interest. Texture features are used to quantify the heterogeneity of the region of interest in terms of grayscale values. For example, a homogeneous structure or tissue would show similar gray values while heterogenous structures would exhibit high differences in gray levels. Examples for this feature class include the Gray Level Co-occurrence Matrix (GLCM), Gray Level Run Length Matrix (GLRLM), or Gray Level Size Zone Matrix (GLSZM). Lastly, wavelet features are calculated from 
- Table 1 Radiomics feature classes and selected examples.

- Tab. 1 Klassen der „Radiomics“-Eigenschaften und ausgewählte Beispiele.

\begin{tabular}{|l|l|l|}
\hline Feature class & Description & Examples \\
\hline $\begin{array}{l}\text { Tumor-intensity based features } \\
\text { (first order statistics) }\end{array}$ & $\begin{array}{l}\text { Histogram-based quantifications of all voxel } \\
\text { intensity values of ROI }\end{array}$ & Minimum, maximum, mean, median, range, etc. \\
\hline Shape features & Used to describe geometric properties of ROI & Volume, surface area, sphericity, maximum diameter, elongation, etc. \\
\hline Texture features & Used to quantify heterogeneity of ROI & $\begin{array}{l}\text { Gray Level Co-occurrence Matrix (GLCM), Gray Level Run Length } \\
\text { Matrix (GLRLM), Gray Level Size Zone Matrix (GLSZM), etc. }\end{array}$ \\
\hline Wavelet features & $\begin{array}{l}\text { Calculated from wavelet decompositions of } \\
\text { original imaging }\end{array}$ & Fourier, Gabor, Haar wavelet transforms \\
\hline
\end{tabular}

wavelet decompositions of the original imaging [16, 23, 24]. A wavelet transformation is a form of mathematical filter that results in decompositions according to the scale and orientation of the initial image. - Table 1 gives an overview of these feature classes and provides relevant examples for each class.

Not all resulting features are equally useful for statistical correlation with clinical endpoints, as there may be redundancies or only a weak association with the classification task [18]. Therefore, to identify which radiomic features should be used as imaging biomarkers for the aimed task, feature selection has to be performed to reduce the dimensionality of the feature space [25]. Multiple studies have compared different feature selection approaches and have thereby shown that feature selection is critical for the development of accurate radiomics models [23, 26, 27]. The last but most challenging step is the development of a model that integrates radiomics data with clinical data to establish models for tumor classification or therapy prediction. This is usually achieved by a regression model for scalar problems (e. g., survival) or a classification model and can be greatly enhanced by machine learning techniques [28].

\section{Addressing Limitations of Radiomics}

A major issue that remains to be solved in the field of radiomics is the reproducibility of results. Many radiomics studies have only used the split-sample approach, meaning that monocentric datasets are split into training and validation datasets before feature extraction [29]. Such models usually show low performance when applied to an independent cohort [30]. A lack of harmonization of data acquisition is still an important drawback for highthroughput data methodologies such as radiomics, since different CT scanner manufacturers, scanning protocols, contrast media, and image reconstruction methods impact image features. Further validation and generalization of results requires large, multicentric datasets that incorporate imaging data and clinical data of good quality to allow for the creation of robust models [30]. Moreover, Ninatti et al. found in their recent review of radiomics approaches in lung cancer that no reliable radiomic features could be identified between different studies [29]. To allow for radiomics approaches to be introduced into clinical practice, further standardization is still required.

\section{Radiogenomics in lung cancer: Predicting the tumor genotype}

As mentioned, therapy of advanced non-small cell lung cancer has changed considerably due to the identification of numerous molecular and genomic markers, making molecular pathological testing part of the clinical routine for NSCLC patients, with mutations in the EGF-receptor, tyrosine kinase receptor ALK, and the oncogenes ROS1 and BRAF usually being investigated since potent therapeutics for these targets are in use [31]. In addition, programmed-death-1 (PD-1)/programmed-death ligand (PD-L1) inhibitors are another important group of novel targeted therapeutics for which testing is routinely performed [31]. These immune checkpoint inhibitors have had a significant impact on patient outcome if no other targetable molecular alteration can be found.

Identification of these biomarkers using radiomics approaches has proven to be promising as it could reduce the need for biopsies or could help track changes in the tumor mutation status over time without the need for re-biopsy. The integration of medical imaging data derived by radiomics and genomic data is often referred to as radiogenomics [32]. The number of publications regarding the identification of these relevant biomarkers in NSCLC patients is increasing ( $\$$ Table 2 ), and several reviews have focused on the comparison of these studies, usually comparing the reliability of the developed models by measuring the area under the curve (AUC). Prediction of EGFR status has seen the highest number of publications in accordance with the relatively high prevalence of mutations in NSCLC patients ( $\downarrow$ Table 2 ) and recent papers have shown good results, with the highest AUC values achieved by combinations of radiomics data, visual qualitative CT features, Al approaches using convolutional neural networks, positron emission tomography parameters, and clini$\mathrm{cal} /$ pathological features [29]. Results in the validation cohorts in these studies ranged from AUCs of 0.73 [33] to 0.95 [34] ( $>$ Table 3).

Only a small number of studies investigating ALK rearrangements have been published and even fewer for PD-L1 expression and the rarer ROS1 and BRAF mutations. These studies showed promising results, for example Yamamoto et al. showed a sensitivity of $83.3 \%$, a specificity of $77.9 \%$, and an accuracy of $78.8 \%$ for 
the prediction model of ALK aberrations [35], and jiang et al. developed a model with an AUC of 0.97 and 0.91 for the prediction of PD-L1 expression rates of $\geq 1 \%$ and $\geq 50 \%$, respectively [36]. These positive outcomes make radiogenomics a promising area for further research since the individual prediction of tumor mutation status solely based on imaging has not been achieved and is not ready for routine use in patients.

- Table 2 Number of publications in the field of radiogenomics in NSCLC.

- Tab.2 Anzahl der Publikationen im Bereich der „Radiogenomics“ bei nichtkleinzelligem Lungenkrebs.

\begin{tabular}{|l|l|l|}
\hline Molecular marker & $\begin{array}{l}\text { Frequency in } \\
\text { patients [3, 10] }\end{array}$ & $\begin{array}{l}\text { Number } \\
\text { of publications }\end{array}$ \\
\hline $\begin{array}{l}\text { EGFR } \\
\text { - Overall }\end{array}$ & $\begin{array}{l}10-20 \% \\
\text { - Exon 19 deletion }\end{array}$ & $\sim 45 \%$ \\
\hline $\begin{array}{l}\text { - Exon 21 L858R } \\
\text { mutation }\end{array}$ & $\sim 40 \%$ & $20+$ \\
\hline - Others & $\sim 15 \%$ & \\
\hline ALK & $2-5 \%$ & 5 \\
\hline ROS1 & $1-4 \%$ & 1 \\
\hline BRAF & $\sim 2 \%$ & 0 \\
\hline PD-L1 expression & $30-40 \%$ & 2 \\
\hline - $1 \%$ & $30-40 \%$ & \\
\hline - 1-49\% & $\sim 30 \%$ & \\
\hline - $50 \%$ & & \\
\hline
\end{tabular}

\section{Multi-omics approaches for response prediction in lung cancer and therapy monitoring}

A deeper understanding of the tumor biology of lung cancer is emerging as increasing attention is given to the heterogeneity and microenvironment of tumors. Even though the identification of the described molecular biomarkers as therapy targets has had a considerable impact on patient outcome, not all patients with corresponding molecular alterations benefit from targeted therapies. Moreover, patients initially benefitting from these therapies often experience recurrence or (hyper-)progression due to acquired therapy resistance of the tumors [37, 38]. Neoplastic transformation results from the accumulation of genetic and epigenetic alterations, leading to a variation of different genetic alterations within the resulting macroscopic tumor. In the example of lung adenocarcinomas, two or more histopathological subtypes have been described with regions with different degrees of differentiation, proliferation, vascularity, inflammation, and invasiveness $[39,40]$. Other factors contributing to the heterogeneity of tumors are epigenetic alterations and the tumor microenvironment [41]. The resulting manifestation of tumor heterogeneity within tumors, in the microenvironment, and between patients has been found to contribute to differences in survival and tumor recurrence as cells that are not susceptible to the administered therapy can replace cells that have been successfully destroyed [42]. Additionally, the accumulation of mutations is a dynamic process that does not stop at the time of initial diagnosis but may lead to resistance mechanisms being acquired during the course of therapy [43]. In clinical practice, this leads to the need for tissue re-biopsy at the time of tumor progression or recurrence from progressing lesions.

- Table 3 Summary of selected studies presented in this review.

- Tab.3 Zusammenfassung der Ergebnisse der präsentierten Studien.

\begin{tabular}{|c|c|c|c|}
\hline Authors & Approach & Modality & Results (in validation cohorts) \\
\hline Zhao et al. [33] & $\begin{array}{l}\text { Prediction model for EGFR gene mutation status in } \\
\text { NSCLC patients }\end{array}$ & CT & $\mathrm{AUC}=0.73$ \\
\hline Jiang et al. [34] & $\begin{array}{l}\text { Prediction model for EGFR gene mutation status in } \\
\text { NSCLC patients }\end{array}$ & $\mathrm{PET} / \mathrm{CT}$ & $\mathrm{AUC}=0.95$ \\
\hline Yamamoto et al. [35] & Model for prediction of ALK aberrations & CT & $\begin{array}{l}\text { Sensitivity } 83.3 \% \text {, specificity } 77.9 \% \text {, accuracy } \\
78.8 \%\end{array}$ \\
\hline Jiang et al. & $\begin{array}{l}\text { Model for prediction of PD-L1 expression rates of } \geq 1 \% \\
\text { or } \geq 50 \%\end{array}$ & $\mathrm{PET} / \mathrm{CT}$ & $\begin{array}{l}\geq 1 \%: A \cup C=0.97 \\
\geq 50 \%: A \cup C=0.91\end{array}$ \\
\hline Xu et al. [45] & $\begin{array}{l}\text { Model to stratify patients into low and high mortality- } \\
\text { risk groups strongly correlating with 2-year overall } \\
\text { survival }\end{array}$ & CT & $\mathrm{AUC}=0.74$ \\
\hline Mu et al. [46] & $\begin{array}{l}\text { Response prediction of advanced NSCLC patients to } \\
\text { immunotherapy }\end{array}$ & $\mathrm{PET} / \mathrm{CT}$ & $\begin{array}{l}\text { AUC }=0.83 \text { (retrospective validation) } \\
A \cup C=0.81 \text { (prospective validation) }\end{array}$ \\
\hline Cucchiara et al. [49] & $\begin{array}{l}\text { Integrating liquid biopsy and radiomics to monitor } \\
\text { clonal heterogeneity in EGFR-positive NSCLC }\end{array}$ & $\begin{array}{l}\mathrm{CT} \\
\text { (liquid biopsy) }\end{array}$ & $R^{2}=0.447, p<0.001$ \\
\hline
\end{tabular}


Radiomics and artificial intelligence-based analysis might offer a noninvasive alternative as it allows for the assessment of the entire tumor volume and can be applied to every radiological follow-up examination, and in theory to every tumor manifestation throughout the body. To further improve the success of targeted therapies, it will be crucial to find better ways to take tumor heterogeneity into account to predict therapy response and monitor tumor phenotypes during therapy. Some studies have already shown the feasibility of predicting tumor response using radiomics approaches [44] ( et al. describe a model that predicts patient outcome by stratifying patients into low and high mortality-risk groups that strongly correlate with 2-year overall survival $(H R=6.16,95 \% \mathrm{Cl}$ $[2.17,17.44], p<0.001)$, achieving an AUC of $0.74(p<0.05)$ for their model [45].

In addition to structural radiomics approaches, functional imaging can also be analyzed using machine-learning approaches. Lung cancer patients receive 18FDG-PET/CT examinations in the clinical routine to assess tumor glucose metabolism as a parameter for tumor activity. Studies have shown that PET-based radiomics can predict clinical outcomes [30]. For example, Mu et al. developed a model identifying NSCLC patients, who are most likely to benefit from immunotherapy with AUC values of 0.86 (95\% Cl 0.79-0.94), 0.83 (95\% Cl 0.71-0.94), and 0.81 (95\% $\mathrm{Cl} 0.68-0.92)$ in the training, retrospective test, and prospective test cohorts, respectively [46].

Integration of structural and functional imaging biomarkers in a multi-omics approach could lead to further improvement of tumor phenotyping with the inclusion of formerly uncorrelated data from new sources [47]. One such example of a new data source is the quickly emerging field of liquid biopsies, i. e., peripheral blood samples. In lung cancer, liquid biopsies offer the possibility to longitudinally extract genetic information from tumors without the need for tissue (re-)biopsy. The genetic information is derived from tumor-specific cfDNA that originates from perishing or circulating tumor cells, giving an overview of all genetic alterations of the tumor [41], thus offering another way to take tumor heterogeneity into account, potentially allowing for therapy response prediction. Janke et al., for example, report a highly significant marker panel indicating therapeutic response $\left(R^{2}=0.78, R^{2}=0.71\right.$, and $\left.R^{2}=0.71\right)$ in patients with advanced nonsmall cell lung cancer receiving chemotherapy or targeted therapies [48]. Studies have already established multi-parametric approaches in smaller data sets. Cucchiara et al. show that the combination of radiomics analyses and liquid biopsy results can be used to monitor mutation status in NSCLC patients over the course of treatment with promising performance on predicting the presence of EGFR mutation status $\left(R^{2}=0.447, p<0.001\right)$ [49].

\section{Conclusion}

The use of radiomics in combination with artificial intelligencebased approaches allows for the identification of novel imaging biomarkers. In non-small cell lung cancer patients, radiogenomics describes the combination of radiomic data with tumor genome mutation status, which is a promising approach for the identifica- tion of therapy targets, such as EGFR mutations, ALK rearrangements, or PD-L1 expression rates, with several prediction models showing encouraging results. Furthermore, radiomics could help to solve the pressing clinical need for early assessment and prediction of therapy response, as not all patients with corresponding molecular alterations benefit from targeted therapies and in many cases tumor recurrence is observed over time. To reach this goal, advanced tumor phenotyping is required, which could be achieved by integrating structural and functional imaging biomarkers with clinical data sources, such as genomics approaches using liquid biopsy results, in a multi-omics approach. However, to allow radiomics- and artificial intelligence-based approaches to be introduced into clinical practice, further standardization using large, multi-center and multi-vendor datasets is required.

\section{Conflict of Interest}

The authors declare that they have no conflict of interest.

\section{Literature}

[1] Sung H, Ferlay J, Siegel RL et al. Global cancer statistics 2020: GLOBOCAN estimates of incidence and mortality worldwide for 36 cancers in 185 countries. CA Cancer J Clin 2021; 71: 209-249

[2] Gerber DE, Gandhi L, Costa DB. Management and future directions in non-small cell lung cancer with known activating mutations. Am Soc Clin Oncol Educ Book 2014: e353-e365

[3] Howlader NNA, Krapcho M, Miller D et al. (eds). SEER Cancer Statistics Review, 1975-2017, National Cancer Institute. 2020. [Available from: https://seer.cancer.gov/csr/1975_2017/

[4] Postmus PE, Kerr KM, Oudkerk M et al. Early and locally advanced nonsmall-cell lung cancer (NSCLC): ESMO Clinical Practice Guidelines for diagnosis, treatment and follow-up. Ann Oncol 2017; 28 (Suppl. 4): iv1-iv21

[5] Villalobos P, Wistuba II. Lung Cancer Biomarkers. Hematol Oncol Clin North Am 2017; 31: 13-29

[6] Hanna N, Johnson D, Temin S et al. Systemic Therapy for Stage IV NonSmall-Cell Lung Cancer: American Society of Clinical Oncology Clinical Practice Guideline Update. J Clin Oncol 2017; 35: 3484-3515

[7] Girard N. Optimizing outcomes in EGFR mutation-positive NSCLC: which tyrosine kinase inhibitor and when? Future Oncol 2018; 14: 1117-1132

[8] Shaw AT, Yeap BY, Mino-Kenudson M et al. Clinical features and outcome of patients with non-small-cell lung cancer who harbor EML4-ALK. J Clin Oncol 2009; 27: 4247-4253

[9] Rosell R, Carcereny E, Gervais R et al. Erlotinib versus standard chemotherapy as first-line treatment for European patients with advanced EGFR mutation-positive non-small-cell lung cancer (EURTAC): a multicentre, open-label, randomised phase 3 trial. The Lancet Oncology 2012; 13: $239-246$

[10] Maemondo M, Inoue A, Kobayashi K et al. Gefitinib or chemotherapy for non-small-cell lung cancer with mutated EGFR. N Engl J Med 2010; 362: $2380-2388$

[11] Solomon B], Mok T, Kim DW et al. First-line crizotinib versus chemotherapy in ALK-positive lung cancer. N Engl J Med 2014; 371: 2167-2177

[12] Peters S, Camidge DR, Shaw AT et al. Alectinib versus Crizotinib in Untreated ALK-Positive Non-Small-Cell Lung Cancer. N Engl ] Med 2017; 377: 829-838

[13] Sorber L, Zwaenepoel K, Deschoolmeester V et al. Circulating cell-free nucleic acids and platelets as a liquid biopsy in the provision of personalized therapy for lung cancer patients. Lung Cancer 2017; 107: 100-107 
[14] Coroller TP, Bi WL, Huynh E et al. Radiographic prediction of meningioma grade by semantic and radiomic features. PloS one 2017; 12: e0187908

[15] Litière $S$, Collette $S$, de Vries EG et al. RECIST - learning from the past to build the future. Nat Rev Clin Oncol 2017; 14: 187-192

[16] Aerts H], Velazquez ER, Leijenaar RT et al. Decoding tumour phenotype by noninvasive imaging using a quantitative radiomics approach. Nat Commun 2014; 5: 4006

[17] Court LE, Rao A, Krishnan S. Radiomics in cancer diagnosis, cancer staging, and prediction of response to treatment. Translational Cancer Research 2016; 5: 337-339

[18] Avanzo M, Stancanello J, El Naqa I. Beyond imaging: The promise of radiomics. Phys Med 2017; 38: 122-139

[19] van Velden FH, Kramer GM, Frings $\vee$ et al. Repeatability of Radiomic Features in Non-Small-Cell Lung Cancer [(18)F]FDG-PET/CT Studies: Impact of Reconstruction and Delineation. Mol Imaging Biol 2016; 18: 788-795

[20] Da-Ano R, Visvikis D, Hatt M. Harmonization strategies for multicenter radiomics investigations. Phys Med Biol 2020; 65: 24tr02

[21] Shinohara RT, Sweeney EM, Goldsmith J et al. Statistical normalization techniques for magnetic resonance imaging. Neuroimage Clin 2014; 6 : 9-19

[22] Rios Velazquez E, Aerts HJWL, Gu Y et al. A semiautomatic CT-based ensemble segmentation of lung tumors: Comparison with oncologists' delineations and with the surgical specimen. Radiotherapy and Oncology 2012; 105: 167-173

[23] Parmar C, Grossmann P, Bussink J et al. Machine Learning methods for Quantitative Radiomic Biomarkers. Scientific reports 2015; 5: 13087

[24] Mayerhoefer ME, Materka A, Langs G et al. Introduction to Radiomics. Journal of Nuclear Medicine 2020. doi:10.2967/jnumed.118.222893

[25] Guyon I, Elisseeff A. An introduction to variable and feature selection. J Mach Learn Res 2003; 3: 1157-1182

[26] Parmar C, Grossmann P, Rietveld D et al. Radiomic Machine-Learning Classifiers for Prognostic Biomarkers of Head and Neck Cancer. Frontiers in oncology 2015; 5: 272

[27] Leger S, Zwanenburg A, Pilz K et al. A comparative study of machine learning methods for time-to-event survival data for radiomics risk modelling. Scientific reports 2017; 7: 13206

[28] Langs G, Röhrich S, Hofmanninger J et al. Machine learning: from radiomics to discovery and routine. Radiologe 2018; 58 (Suppl. 1): 1-6

[29] Ninatti G, Kirienko M, Neri E et al. Imaging-Based Prediction of Molecular Therapy Targets in NSCLC by Radiogenomics and Al Approaches: A Systematic Review. Diagnostics (Basel) 2020; 10: 6

[30] Wu G, Jochems A, Refaee T et al. Structural and functional radiomics for lung cancer. Eur J Nucl Med Mol Imaging 2021; 48: 3961-3974

[31] Planchard D, Popat S, Kerr K et al. Metastatic non-small cell lung cancer: ESMO Clinical Practice Guidelines for diagnosis, treatment and followup. Annals of Oncology 2018; 29: iv192-iv237

[32] Lo Gullo R, Daimiel I, Morris EA et al. Combining molecular and imaging metrics in cancer: radiogenomics. Insights Imaging 2020; 11: 1
[33] Zhao W, Wu Y, Xu Yn et al. The Potenzial of Radiomics Nomogram in Non-invasively Prediction of Epidermal Growth Factor Receptor Mutation Status and Subtypes in Lung Adenocarcinoma. Frontiers in oncology 2020; 9: 1485

[34] Jiang M, Zhang Y, Xu J et al. Assessing EGFR gene mutation status in nonsmall cell lung cancer with imaging features from PET/CT. Nucl Med Commun 2019; 40: 842-849

[35] Yamamoto S, Korn RL, Oklu R et al. ALK molecular phenotype in nonsmall cell lung cancer: $\mathrm{CT}$ radiogenomic characterization. Radiology 2014; 272: 568-576

[36] Jiang M, Sun D, Guo Y et al. Assessing PD-L1 Expression Level by Radiomic Features From PET/CT in Nonsmall Cell Lung Cancer Patients: An Initial Result. Acad Radiol 2020; 27: 171-179

[37] Lin JJ, Riely G], Shaw AT. Targeting ALK: Precision Medicine Takes on Drug Resistance. Cancer Discov 2017; 7: 137-155

[38] Westover D, Zugazagoitia J, Cho BC et al. Mechanisms of acquired resistance to first- and second-generation EGFR tyrosine kinase inhibitors. Ann Oncol 2018; 29 (Suppl. 1): i10-i19

[39] Lee G, Lee HY, Park H et al. Radiomics and its emerging role in lung cancer research, imaging biomarkers and clinical management: State of the art. Eur J Radiol 2017; 86: 297-307

[40] Motoi N, Szoke J, Riely G] et al. Lung adenocarcinoma: modification of the 2004 WHO mixed subtype to include the major histologic subtype suggests correlations between papillary and micropapillary adenocarcinoma subtypes, EGFR mutations and gene expression analysis. Am J Surg Pathol 2008; 32: $810-827$

[41] Voigt W, Manegold C, Pilz L et al. Beyond tissue biopsy: a diagnostic framework to address tumor heterogeneity in lung cancer. Curr Opin Oncol 2020; 32: $68-77$

[42] Oser MG, Niederst M], Sequist LV et al. Transformation from non-smallcell lung cancer to small-cell lung cancer: molecular drivers and cells of origin. The Lancet Oncology 2015; 16: e165-e172

[43] Gerlinger M, Rowan A], Horswell S et al. Intratumor heterogeneity and branched evolution revealed by multiregion sequencing. N Engl J Med 2012; 366: 883-892

[44] Avanzo M, Stancanello J, Pirrone G et al. Radiomics and deep learning in lung cancer. Strahlenther Onkol 2020; 196: 879-887

[45] Xu Y, Hosny A, Zeleznik R et al. Deep Learning Predicts Lung Cancer Treatment Response from Serial Medical Imaging. Clinical cancer research : an official journal of the American Association for Cancer Research 2019; 25: 3266-3275

[46] Mu W, Tunali I, Gray JE et al. Radiomics of (18)F-FDG PET/CT images predicts clinical benefit of advanced NSCLC patients to checkpoint blockade immunotherapy. Eur J Nucl Med Mol Imaging 2020; 47: 1168-1182

[47] Gillies RJ, Kinahan PE, Hricak H. Radiomics: Images Are More than Pictures, They Are Data. Radiology 2016; 278: 563-577

[48] Janke F, Bozorgmehr F, Wrenger $S$ et al. Novel Liquid Biomarker Panels for A Very Early Response Capturing of NSCLC Therapies in Advanced Stages. Cancers (Basel) 2020; 12: 4

[49] Cucchiara F, Del Re M, Valleggi S et al. Integrating Liquid Biopsy and Radiomics to Monitor Clonal Heterogeneity of EGFR-Positive Non-Small Cell Lung Cancer. Frontiers in oncology 2020; 10: 2664 